\title{
Effects of endoplasmic reticulum stress-mediated CREB3L1 on apoptosis of glioma cells
}

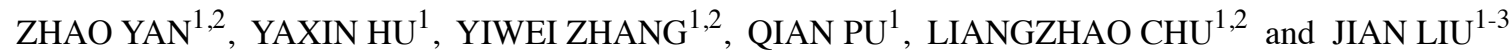 \\ ${ }^{1}$ Department of Clinical Medicine, Guizhou Medical University, Guiyang, Guizhou 550004; \\ ${ }^{2}$ Department of Neurosurgery, The Affiliated Hospital of Guizhou Medical University, Guiyang, Guizhou 550001; \\ ${ }^{3}$ Department of Neurosurgery, Guizhou Provincial People's Hospital, Guiyang, Guizhou 550002, P.R. China
}

Received October 20, 2021; Accepted January 24, 2022

DOI: $10.3892 / \operatorname{mco} .2022 .2516$

\begin{abstract}
The association between endoplasmic reticulum stress (ERS) and apoptosis has been extensively studied. Cyclic adenosine monophosphate responsive element binding protein 3 like 1 (CREB3L1) has an important role in the development of glioma. In the present study, the potential association between ERS-induced apoptosis and CREB3L1 and its clinical implications were investigated. From a total of 30 cases, brain gliomas with different pathological grades surgically resected at the Department of Neurosurgery of the Affiliated Hospital of Guizhou Medical University (Guiyang, China) between January 2018 and January 2019 were collected. The expression of CREB3L1 and ERS-related proteins in gliomas with different degrees of malignancy was detected by immunohistochemistry. Furthermore, U87-MG glioma cells were cultured in vitro and treated with different concentrations of ERS inducer thapsigargin (TG). The Cell Counting Kit-8 (CCK-8) assay was performed to detect changes in cell activity at different incubation times and drug concentrations. Cell apoptosis was detected by Annexin V-FITC/propidium iodide double staining and the protein expression levels of CREB3L1 and ERS were detected by western blot analysis. Immunohistochemical analysis suggested that the expression levels of CREB3L1, glucose-regulated protein, $78 \mathrm{kDa}$ (GRP78) and C/EBP-homologous protein (CHOP) in World Health Organization (WHO) grade I glioma were higher than those in WHO grade II-IV (all P<0.01). The results of the CCK-8
\end{abstract}

Correspondence to: Professor Jian Liu, Department of Clinical Medicine, Guizhou Medical University, 9 Beijing Road, Yunyan, Guiyang, Guizhou 550004, P.R. China

E-mail: ycayxt@163.com

Abbreviations: ERS, endoplasmic reticulum stress; CREB3L1, cyclic adenosine monophosphate responsive element binding protein 3 like 1; IHC, immunohistochemistry; FCM, flow cytometry; CHOP, $\mathrm{C} / \mathrm{EBP}-$ homologous protein

Key words: endoplasmic reticulum stress, thapsigargin, U87-MG, glioma cells, apoptosis, CREB3L1 assay suggested that the activity of U87-MG glioma cells was significantly decreased after treatment with TG (all $\mathrm{P}<0.05)$, and this effect was time- and drug concentration-dependent. Flow cytometric analysis indicated that the apoptotic rate of the cells was increased, which was significant when the concentration of TG was $0.1 \mu \mathrm{mol} / \mathrm{l}(\mathrm{P}<0.01)$. Furthermore, the protein expression of CREB3L1, GRP78 and CHOP in glioma cells treated with TG was increased $(\mathrm{P}<0.05)$. However, the expression level of $\mathrm{Bcl}-2$ decreased $(\mathrm{P}<0.05)$. In conclusion, ERS may reduce the cell proliferative activity and promote apoptosis through mediating CREB3L1 expression. CREB3L1 may be a novel potential target for glioma therapy.

\section{Introduction}

Glioma is a central nervous system tumor derived from glial cells. Malignant primary brain tumor remains one of the most difficult cerebral neoplasms to treat. In recent years, despite significant progress in treatments such as surgery or combined chemoradiotherapy, the prognosis of patients with glioma is still discouraging and the 5-year survival rate is $<35 \%$ (1). Glioma, particularly glioblastoma, has a high degree of malignancy and the survival time of patients after diagnosis is $<15$ months. New drugs, delivery systems, immunotherapy and other technical means are being studied to identify suitable therapeutic targets in abnormal molecular pathways (2).

Endoplasmic reticulum (ER) is a network structure of cytoplasmic parts. Its main functions include protein synthesis, modification and processing. When cells receive a variety of strong stimuli, such as nutrient deficiency, $\mathrm{Ca}^{2+}$ metabolism imbalance, toxin stimulation or persistent oxidative stress, the homeostasis of cells is broken and ER stress (ERS) is then activated to restore homeostasis. However, when ERS persists, the damaged ER is engulfed and degraded (3). Thapsigargin (TG) is a cytotoxic drug that is able to block the ER Ca ${ }^{2+}$ ATPase pump, disrupt $\mathrm{Ca}^{2+}$ homeostasis and initiate ERS (4).

Cyclic adenosine monophosphate responsive element binding protein 3 like 1 (CREB3L1) protein is a BZIP-type transcription factor in the CREB/ATF family of cyclic adenylate response element binding proteins. ERS is a necessary condition for the activation of CREB3L1, which is highly expressed in osteoblasts of bone tissue and astrocytes of the central nervous system (5). CREB3L1 has been indicated to 
be abnormally expressed in a variety of tumors and has an important role (6-8).

In the present study, the expression of CREB3L1 in glioma tissues of different grades was investigated and the effect of ERS on the expression of CREB3L1 on the activity and apoptosis of glioma cells was then investigated in order to provide a theoretical basis for the diagnosis and treatment of glioma.

\section{Materials and methods}

Glioma samples. Formalin-fixed and paraffin-embedded tissue blocks were collected from 30 patients diagnosed at the Department of Pathology of Guizhou Medical University (Guiyang, China) between January 2018 and January 2019, including 8 cases of World Health Organization (WHO) grade I, 7 cases of WHO grade II, 7 cases of WHO grade III and 8 cases of WHO grade IV. The experimental protocol was established according to the ethical guidelines of the Helsinki Declaration and approved by the Human Ethics Committee of Guizhou Medical University (Guiyang, China) and written informed consent was obtained from each patient. The demographic and clinicopathological data of the patients are provided in Table I.

Cell culture and main materials. U87-MG cells (cat. no. CL-0238) were obtained from Procell Life Science \& Technology Co., Ltd. In addition, the identity of the cell line was verified by short tandem repeat profiling. Genomic DNA was extracted from U87-MG cells using a PureLink ${ }^{\circledR}$ Genomic DNA Mini Kit (cat. no. K182001; Thermo Fisher Scientific, Inc.). A PowerPlex ${ }^{\circledR} 18 \mathrm{D}$ kit (cat. no. DC1802; Promega Corporation) was used for amplification and an ABI3500 Genetic Analyzer (cat. no. 3500; Applied Biosystems; Thermo Fisher Scientific, Inc.) was used for detection. The results indicated that no cross-contamination of human cells was present in the U87-MG cell line. U87-MG glioma cells were cultured in DMEM supplemented with 10\% FBS (Gibco; Thermo Fisher Scientific, Inc.) and antibiotics $(100 \mu \mathrm{g} / \mathrm{ml}$ penicillin and $100 \mu \mathrm{g} / \mathrm{ml}$ streptomycin) in a humidified atmosphere with $5 \% \mathrm{CO}_{2}$ at $37^{\circ} \mathrm{C}$. TG was purchased from Beyotime Institute of Biotechnology, a Cell Counting Kit-8 (CCK-8) assay kit was purchased from APeXBIO Technology LLC and an Annexin V-FITC/PI double staining cell apoptosis kit was purchased from Nanjing KeyGen Biotech Co., Ltd. After $24 \mathrm{~h}$ of incubation, samples were collected for flow cytometry (FCM) and western blot analysis.

Immunohistochemistry (IHC). Samples were sectioned at $3 \mu \mathrm{m}$ thickness with a conventional pathological microtome, and then the slices were roasted, dewaxed and hydrated. Antigen retrieval was performed in a pressure cooker using Tris-EDTA ( $\mathrm{pH}$ 9.0). Sections were washed in PBS and treated with $0.3 \% \mathrm{H}_{2} \mathrm{O}_{2}$ at room temperature for $15 \mathrm{~min}$. Sections were then blocked in 5\% goat serum (cat. no. C0265; Beyotime Institute of Biotechnology) in PBS at room temperature for $30 \mathrm{~min}$, followed by incubation with primary antibody against CREB3L1 (rabbit; cat. no. 11235-2-AP; 1:100 dilution; ProteinTech Group), glucose-regulated protein, $78 \mathrm{kDa}$ [GRP78, also known as heat shock protein family A (Hsp70) member 5] (rabbit; Bs-1219R; 1:200 dilution; BIOSS) and
C/EBP-homologous protein (CHOP) (rabbit; Bs-20669R; 1:300 dilution; BIOSS) in working solution overnight at $4^{\circ} \mathrm{C}$. Subsequently, the samples were incubated with goat anti-rabbit IgG H\&L/HRP (cat. no. bs-40295G-HRP; dilution, 1:3000; $\mathrm{BIOSS}$ ) at $37^{\circ} \mathrm{C}$ for $30 \mathrm{~min}$. Colorimetric signals were developed using diaminobenzidine substrate (Vector Laboratories, Inc.) followed by counterstaining with hematoxylin, dehydration and sealing with gum. The images, captured by Olympus BX53 biological microscope (Olympus Corporation), were analyzed with the ImagePro Plus 6.0 software package (Media Cybernetics, Inc.) and the expression level of target protein was calculated as the integrated optical density (sum)/area (sum).

CCK-8 assay. U87-MG glioma cells in the logarithmic growth phase were collected and the cell concentration was adjusted to $5 \times 10^{4} / \mathrm{ml}$. The cell suspension ( $100 \mu \mathrm{l} /$ well) was inoculated into 96-well plates and cultured in a $5 \% \mathrm{CO}_{2}$ cell incubator at $37^{\circ} \mathrm{C}$ for $24 \mathrm{~h}$. Subsequently, $10 \mu \mathrm{l} \mathrm{TG}$ was added into the wells to result in different final concentrations $(0.025,0.05$ or $0.1 \mu \mathrm{mol} / \mathrm{l})$, while DMSO was added to the negative control group (final concentration of DMSO, <0.1\%). The culture was continued in the incubator for 6, 12, 24 and $48 \mathrm{~h}$. A total of six wells were selected from each group at each time-point and CCK-8 reagent (10 $\mu \mathrm{l} /$ well) was added to the wells. After $2 \mathrm{~h}$ of incubation, the absorbance value of each well at $450 \mathrm{~nm}$ was detected by a microplate reader.

FCM. An appropriate amount of U87-MG glioma cells in the logarithmic growth phase were inoculated into 6-well plates and treated under the corresponding conditions $(0,0.025$, 0.05 or $0.1 \mu \mathrm{mol} / \mathrm{l}$ ) for $24 \mathrm{~h}$. The cells were then digested with trypsin without EDTA, washed twice with $1 \mathrm{ml}$ PBS (with centrifugation at $754.65 \mathrm{x}$ g for $5 \mathrm{~min}$ at room temperature and $1-5 \times 10^{5}$ cells were collected. The cells were suspended with $500 \mu \mathrm{l}$ Binding Buffer, mixed with $5 \mu \mathrm{l}$ Annexin V-FITC and $5 \mu \mathrm{l}$ propidium iodide (PI) was then added. Following mixing, cells were incubated at room temperature in the dark for 5-15 min. The cells were then analyzed with a flow cytometer (B47905; Beckman Coulter) within $1 \mathrm{~h}$.

Western blot analysis. Glioma cells treated with different concentrations of TG for $24 \mathrm{~h}$ were collected and then were lysed with RIPA lysis buffer and the total protein was extracted. The protein concentration was determined using the BCA method. Total protein ( $20 \mu \mathrm{g}$ per lane) was separated by $10 \%$ SDS-PAGE and then transferred to $0.45 \mu \mathrm{m}$ PVDF membranes (EMD Millipore), followed by blocking with 5\% skimmed milk (Yili) at room temperature for $1 \mathrm{~h}$. The membranes were then incubated with primary antibody [GRP78 (cat. no. bs-1219R; dilution, 1:1,000; BIOSS); CREB3L1 (cat. no. 11235-1-AP; dilution, 1:1,000; ProteinTech Group); Bcl-2 (cat. no. AB112; dilution, 1:1,000; Beyotime Institute of Biotechnology); and CHOP (cat. no. Bs-20669R; dilution, 1:1,000; BIOSS)] at the dilution recommended by the supplier at $4^{\circ} \mathrm{C}$ overnight. The next day, the excess primary antibody was washed off with TBST and the secondary antibody (goat anti-rabbit $\operatorname{IgG}(\mathrm{H}+\mathrm{L})$; cat. no. PMK-014-090M; dilution, 1:5,000; BIOPRIMACY) was added, followed by incubation at room temperature for $2 \mathrm{~h}$. The bands were visualized using enhanced chemiluminescence (ChemiScope; Clinx) and the gray value of the protein 
Table I. Demographic data of the patients by WHO grade.

\begin{tabular}{lccccc}
\hline Characteristic & Total $(\mathrm{n}=30)$ & WHO I $(\mathrm{n}=8)$ & WHO II $(\mathrm{n}=7)$ & WHO III $(\mathrm{n}=7)$ & WHO IV $(\mathrm{n}=8)$ \\
\hline Age, years & $46.5(1.2-85)$ & $46.5(20-73)$ & $47(1.2-75)$ & $46(3.8-66)$ \\
Sex, M/F & $12 / 18$ & $2 / 6$ & $2 / 5$ & $2 / 5$ & $48.5(3-77)$ \\
\hline
\end{tabular}

Values are expressed as the median (interquartile range) or N. WHO, World Health Organization; M, male; F, female.

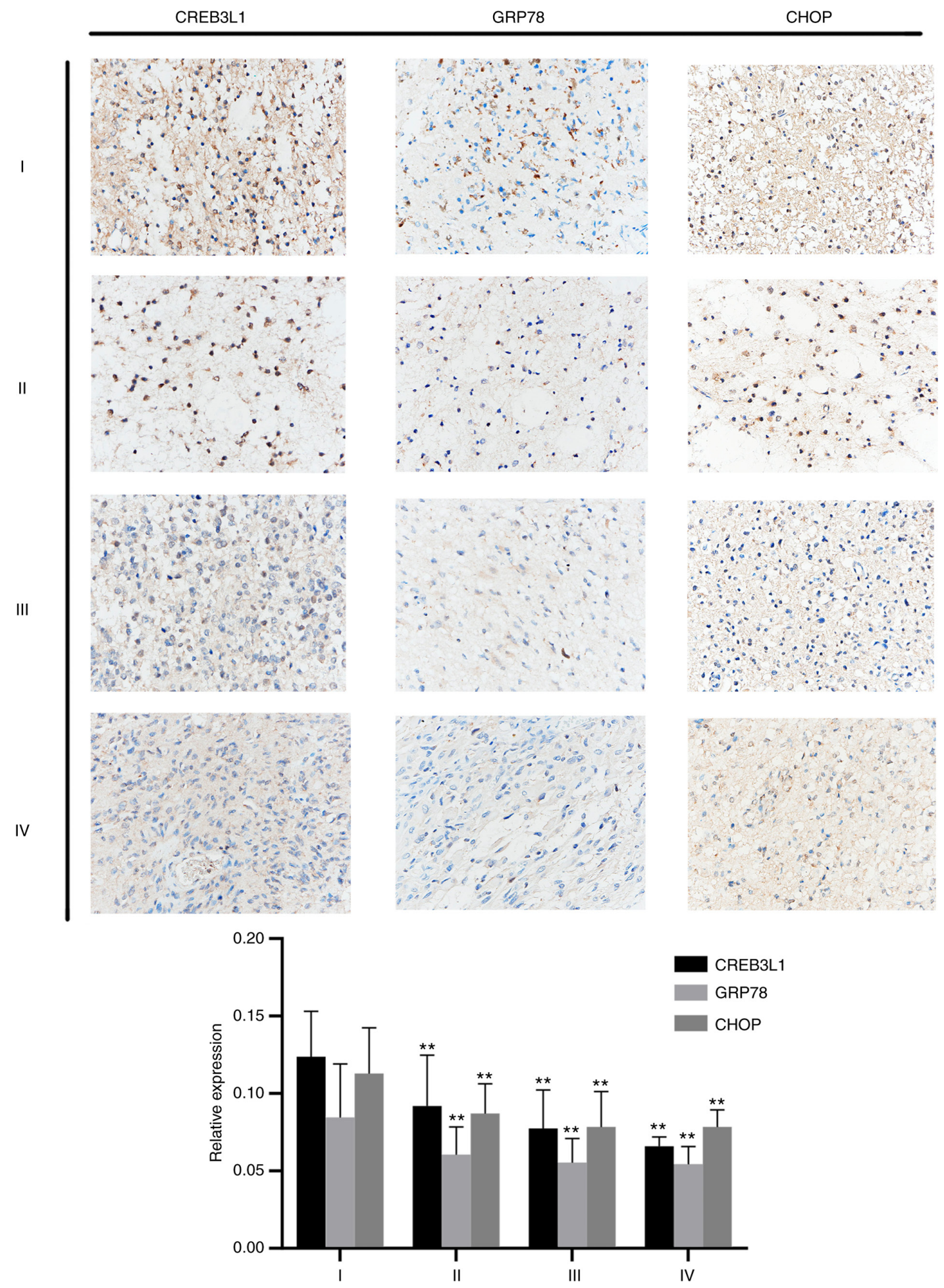

Figure 1. Expression of CREB3L1, GRP78 and CHOP in World Health Organization grade I-IV glioma tissues detected by immunohistochemistry (magnification, $\mathrm{x} 400)$. Values are expressed as the mean \pm standard deviation $(\mathrm{n}=3) .{ }^{* *} \mathrm{P}<0.01$ vs. grade I. CREB3L1, cyclic adenosine monophosphate responsive element binding protein 3 like 1; CHOP, C/EBP-homologous protein; GRP78, glucose-regulated protein, $78 \mathrm{kDa}$. 
was determined with ImageJ v. 1.52 (National Institutes of Health) for quantification.

Statistical analysis. All data were analyzed using SPSS 25.0 statistical software (IBM Corporation). The measurement data conforming to a normal distribution were represented as the mean \pm standard deviation. Multigroup comparisons of the means were performed by one-way ANOVA followed by a Student-Newman-Keuls post-hoc test and the GraphPad Prism 8 software package (GraphPad Software, Inc.) was used to plot the data. $\mathrm{P}<0.05$ was considered to indicate a statistically significant difference.

\section{Results}

CREB3L1 is associated with the grade of glioma. The cohort included 12 males and 18 females with a mean age of 46.5 years (interquartile range, 1.2-85 years). Positive staining for CREB3L1, GRP78 and CHOP in glioma tissue sections was mainly present in the cytoplasm and appeared as brown-yellow granules. As presented in Fig. 1, the expression level of CREB3L1 in WHO I grade glioma was higher than that in WHO Grade II, WHO Grade III and WHO Grade IV glioma (all $\mathrm{P}<0.01$ ). The expression level of GRP78 in WHO I grade was higher than that in WHO grade II, WHO grade III and WHO grade IV glioma (all $\mathrm{P}<0.01$ ); The expression level of CHOP in WHO I grade was higher than that in WHO grade II, WHO grade III and WHO grade IV (all $\mathrm{P}<0.01$ ). These results suggested that CREB3L1 may be related to the development of glioma (Fig. 1).

$T G$ reduces the proliferative activity of glioma cells. The results of the CCK- 8 assay indicated that the proliferative ability of cells treated with TG was significantly lower than that of the blank group. Compared with that in the control group, the activity of U87-MG glioma cells was significantly decreased with the increase of the concentration at 12 , 24 and $48 \mathrm{~h}$ (all $\mathrm{P}<0.05)$. When the concentration of TG was $0.1 \mu \mathrm{mol} / \mathrm{ml}$, compared with that at $6 \mathrm{~h}$, a longer treatment time led to a significantly lower growth activity of U87-MG glioma cells (all $\mathrm{P}<0.01$; Fig. 2). These results indicated that TG inhibited the proliferation of glioma in a concentrationand time-dependent manner.

$T G$ promotes apoptosis of U87-MG glioma cells. In order to further detect the effect of TG on the apoptosis of U87-MG glioma cells, the apoptotic rate of different groups of cells was measured by FCM. An Annexin V-FITC/PI double staining kit was used for the experiment. TG was able to obviously induce apoptosis of glioma U87-MG cells. The apoptosis rate of the control group was $6.69 \pm 2.59 \%$ (Fig. 3A). The apoptosis rate of glioma U87-MG cells increased in a dose-dependent manner with the increase of TG levels $(8.87 \pm 2.32,12.68 \pm 3.51$ and $19.15 \pm 4.88 \%$; Fig. 3B-D). When the drug concentration reached $0.1 \mu \mathrm{mol} / 1$, the difference was statistically significant $(\mathrm{P}<0.01)$. As indicated in Fig. 3E, the apoptotic rate rose with increasing doses of TG.

Effects of ERS on apoptosis-related proteins in glioma cells. Western blot analysis indicated that the expression levels of

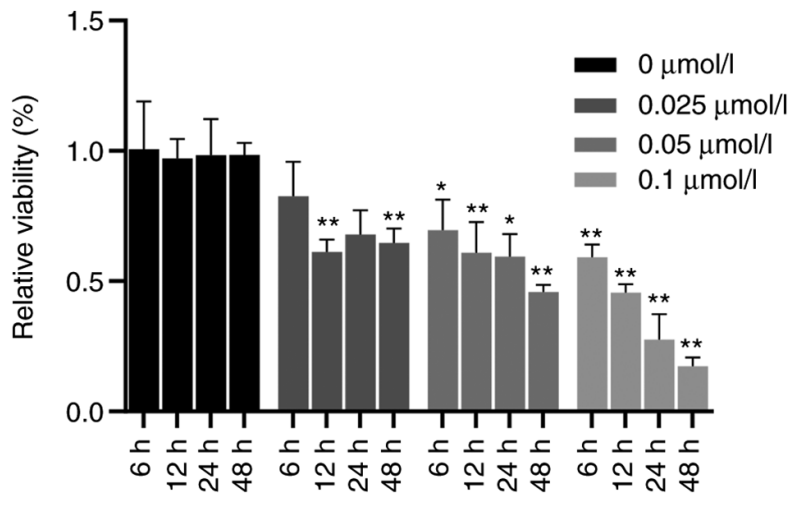

Figure 2. Effects of different concentrations of thapsigargin at different incubation times on the activity of U87-MG glioma cells as determined by a Cell Counting Kit- 8 assay. Values are expressed as the mean \pm standard deviation $(\mathrm{n}=3) .{ }^{*} \mathrm{P}<0.05,{ }^{* *} \mathrm{P}<0.01$ vs. control.

GRP78, CREB3L1 and CHOP in glioma cells increased after ERS, while the expression level of Bcl-2 decreased. When the drug concentration was $0.1 \mu \mathrm{mol} / 1$, the differences from the control group were statistically significant $(\mathrm{P}<0.05$; Fig. 4).

\section{Discussion}

Glioma is a highly complex and malignant tumor type of the central nervous system with a high mortality rate. Due to factors such as the blood-brain barrier and multi-drug resistance, the efficacy of chemotherapy for glioma is limited (9). It is of great significance to further study the molecular mechanisms associated with glioma and its therapeutic drug targets.

The ERS response is the self-protection mechanism of stressed cells. When ERS is induced, tumor cells activate the adaptive mechanism of the unfolded protein response, which is a comprehensive signaling system that may restore ER homeostasis and induce cell apoptosis (10). Apoptosis is a process of programmed cell death under the stimulation of the internal and external environment. It is also an important cause of cell death induced by anti-tumor drugs (11). Apoptosis has a negative regulatory role in the occurrence and development of tumors. The apoptosis genes known to be related to the occurrence and development of tumors mainly include the Bcl-2 family, Caspase family and the P53 gene. Bcl-2 is an anti-apoptotic protein in the Bcl-2 family. Downregulation of Bcl-2 may promote apoptosis of tumor cells, while overexpression of Bcl-2 may inhibit it (12). It has been indicated that when the external stimulation was too strong, the ER induces cell apoptosis through activation of the PKR-like ER kinase (PERK), inositol-requiring enzyme 1 and activating transcription factor 6 (ATF6) pathways (13). ATF6 is a transcription factor that is transferred to the Golgi body in response to ERS, where it is cleaved by site- 1 proteases (S1P) and S2P, activates target gene GRP78 and then indirectly modulates cell apoptosis through CHOP (14). It is well known that CHOP is a key pro-apoptotic molecule that may lead to bodily damage by causing cell cycle arrest and inducing cell death. CHOP is involved in the PERK-ATF4-CHOP signaling pathway during cell apoptosis. Bcl-2 family members are also involved in the process. In addition to the above three signaling pathways, the CREB3L1 transcription factor, similar to ATF6 (15), is 
A

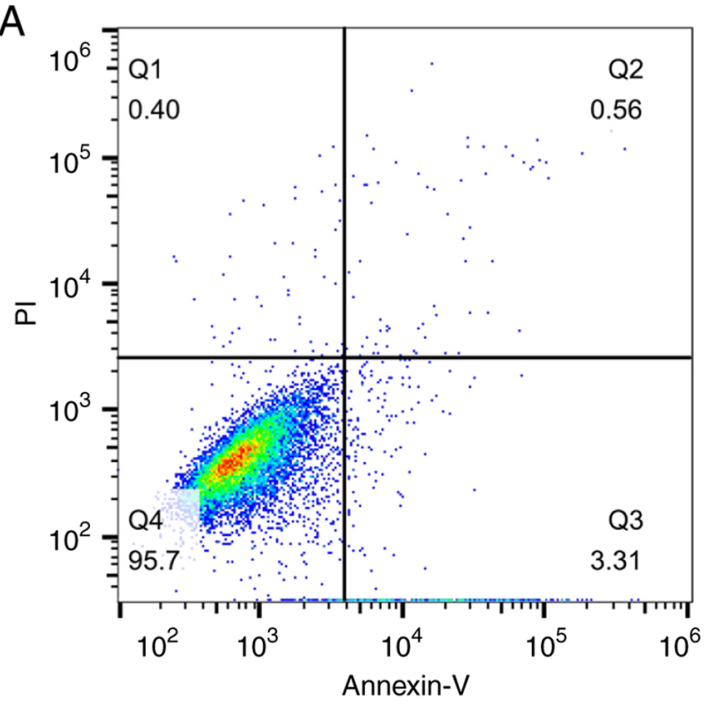

C

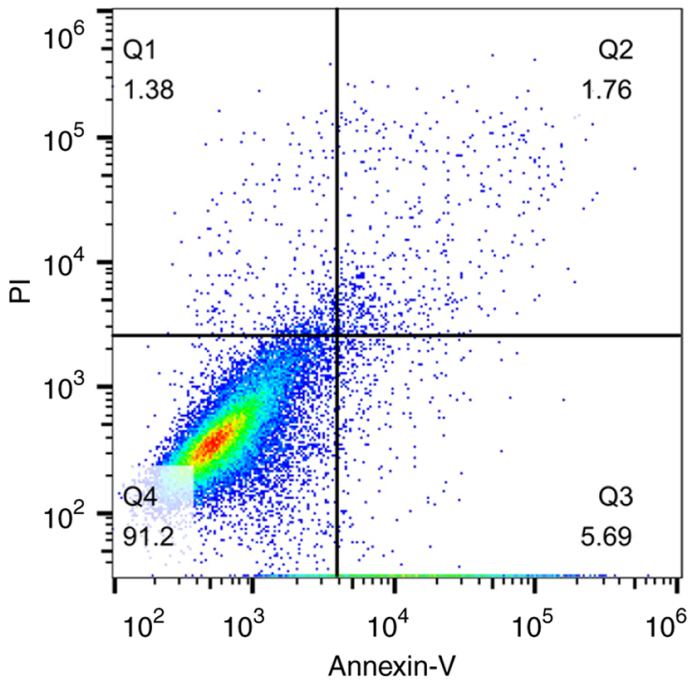

B

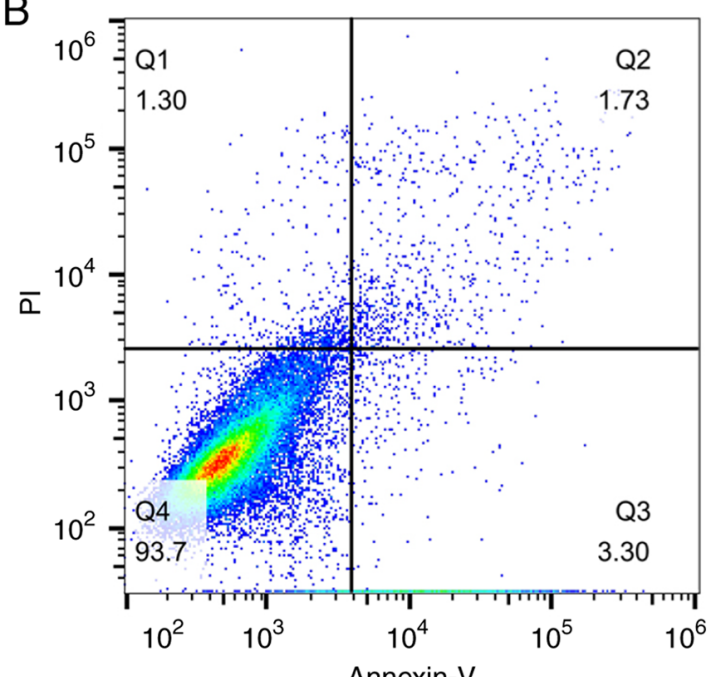

D

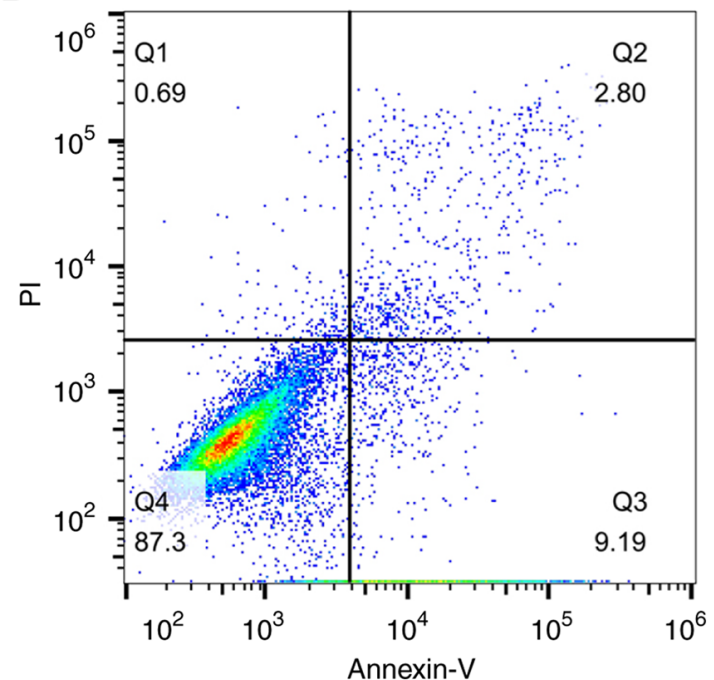

E

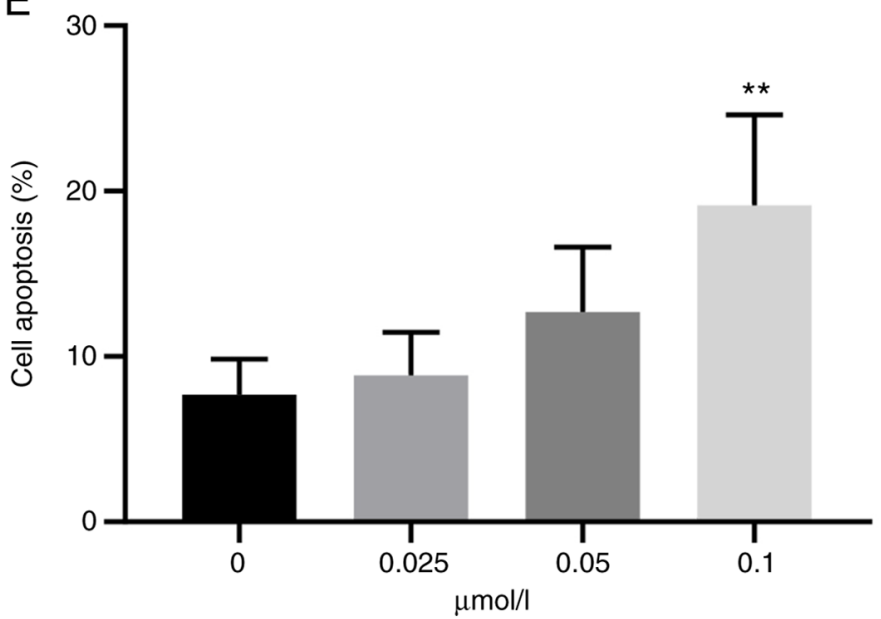

Figure 3. Effects of different concentrations of TG on apoptosis of U87-MG glioma cells. (A) Control group. (B-D) The apoptotic rate of glioma U87-MG cells increased in a dose-dependent manner with the increase of TG levels. The concentration in each group was (A) 0 , (B) $0.025,(\mathrm{C}) 0.05$ and (D) $0.1 \mu$ mol/1 and the apoptotic rates were (B) $8.87 \pm 2.32 \%$, (C) $12.68 \pm 3.51 \%$ and (D) $19.15 \pm 4.88 \%$. (E) Quantified apoptotic rate in each group, indicating that apoptosis was enhanced with the increase of the dose of TG. Values are expressed as the mean \pm standard deviation ( $\mathrm{n}=3$ ). ${ }^{* *} \mathrm{P}<0.01$ vs. control. TG, thapsigargin; PI, propidium iodide; Q, quadrant.

also a type II membrane protein with cytoplasmic N-terminal transcription (16). As a member of the CREB3 family, ERS is necessary to activate CREB3L1. Kondo et al (17) indicated that in the absence of ERS, CREB3L1 (also named OASIS) 


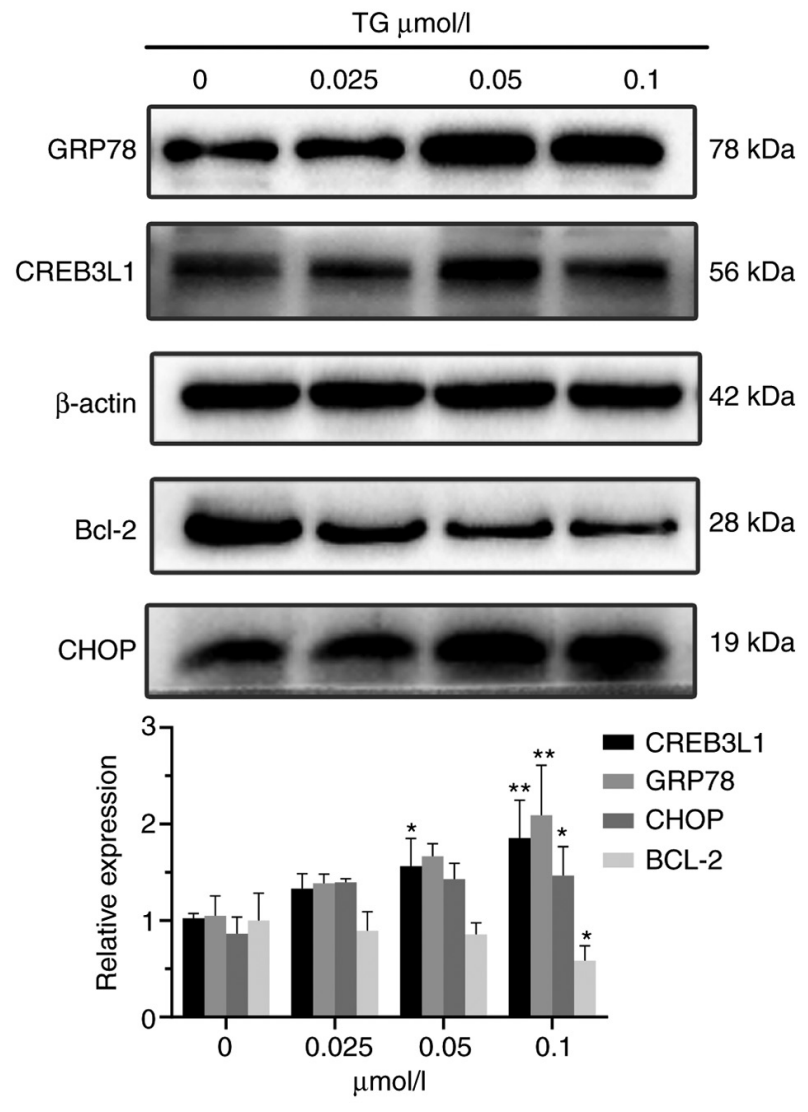

Figure 4. Expression of CREB3L1, GRP78, CHOP and Bcl-2 following incubation with different concentrations of TG. Values are expressed as the mean \pm standard deviation $(n=3) .{ }^{*} \mathrm{P}<0.05,{ }^{* *} \mathrm{P}<0.01$ vs. control. CREB3L1, cyclic adenosine monophosphate responsive element binding protein 3 like 1; CHOP, C/EBP-homologous protein; GRP78, glucose-regulated protein, $78 \mathrm{kDa}$; $\mathrm{TG}$, thapsigargin.

degraded rapidly in C6 glioma cells and its expression level dropped to $10 \%$ within $2 \mathrm{~h}$; however, the degradation rate of CREB3L1 was significantly inhibited by ERS and the expression level of CREB3L1 remained at 40-90\% within $2 \mathrm{~h}$. This suggests that, although CREB3L1 is unstable and easily degraded by proteasomes under normal conditions, they are stabilized by ERS.

TG was originally in public use for the treatment of rheumatic pain, lung disease and female infertility. Recently, it was indicated that it is an effective cytotoxin that may induce cell apoptosis by inhibiting the sarcoplasmic/ER $\mathrm{Ca}^{2+}$ ATPase pump and may be used as a novel type of anti-tumor drug (18). The results of the present study suggested that TG was able to inhibit the cell activity of glioma U87-MG cells in a dose-dependent manner. In order to further verify the promoting effect of TG on the apoptosis of glioma U87-MG cells, FCM was used in the present study to determine the apoptotic rate of cells and the results suggested that TG was able to promote the apoptosis of glioma cells. In a previous study on rheumatoid arthritis, TG induced MH7A cells in a time- and dose-dependent manner (19). TG was able to effectively impair the cell proliferation and survival of MH7A cells. It may be assumed that TG is not only able to cause cell apoptosis when the endoplasmic reticulum stress is high to affect cell activity but also inhibits cell proliferation through non-apoptotic pathways. This mechanism may be worthy of further exploration.
In view of the regulatory effect of ERS on the biological function of glioma cells, the possible regulatory mechanism was discussed in the present study. First, according to IHC analysis, the expression of proteins closely associated with malignant progression of glioma indicated that certain factors may induce CREB3L1 gene mutations, reduce CREB3L1 transcription factor expression, block the pathway of apoptosis and cause excessive proliferation, eventually leading to an increase in the degree of malignancy of glioma (7). These results suggest that CREB3L1 may act as a tumor suppressor gene to promote apoptosis. Next, western blot analysis further confirmed that the expression of ERS-related proteins GRP78 and CHOP increased after TG treatment, indicating that TG may cause ERS, significantly increase the expression level of CREB3L1 and markedly decrease the level of Bcl-2. These results suggested that the apoptosis induced by ERS may be related to CREB3L1. In their study on breast cancer, Raiter et al (20) indicated that CREB3L1 enhanced the expression of GRP78 on the surface of triple-negative breast cells and reduced their ability to migrate and metastasize. Xiao et al (21) reported that among patients with soft-tissue sarcoma, the survival rate of patients with high CREB3L1 expression was significantly higher than that of patients with low CREB3L1 expression and the expression level of CREB3L1 was determined as an independent prognostic factor for survival. In a study on endothelial angiogenesis, CREB3L1 was indicated to be a bona fide functional target of microRNA-146a, regulating angiogenesis (22). In addition, in a study on prostate cancer, inhibition of CREB3L1 expression was observed to promote the proliferation of cancer cells, which may provide novel possibilities for potential therapeutic targets for prostate cancer (23).

In conclusion, the present study indicated that CREB3L1 was highly expressed in glioma tissues and correlated with the degree of malignancy of the tumor. ERS may affect cell proliferation and promote cell apoptosis through mediating CREB3L1 expression. However, certain limitations should be noted. First, it was not possible to evaluate the extent of apoptosis by a second independent method due to a lack of relevant antibodies. Furthermore, the effect estimates in the experiments are based on conventional and prospective observational studies; the experimental methods are relatively traditional and the results are mostly in the form of changes in cell phenotypes. Therefore, as for the specific mechanism through which CREB3L1 affects the occurrence and development of glioma, additional studies are required to further clarify the role of CREB3L1 in glioma and provide a new direction for the diagnosis and treatment of glioma.

\section{Acknowledgements}

Not applicable.

\section{Funding}

This study was funded by the National Natural Science Foundation of China (grant no. 81560409) and the Science Foundation of Guizhou Province of China [grant nos. (2014) 6008 and QianKeHe (2016) support 2905] and the Science and Technology Foundation approved by Guizhou Provincial Health Commission [no. gzwkj (2022-090)]. 


\section{Availability of data and materials}

The data generated and analyzed during the present study are available from the corresponding author on reasonable request.

\section{Authors' contributions}

Conceptualization and methodology: LC, JL and $\mathrm{YH}$. Validation and investigation (including clinical sample collection and other related cell experiments): ZY, YZ and QP. Writing-original draft preparation and writing-review and editing: JL, LC and ZY. Reading and approval of final manuscript: All authors. ZY, JL and YZ checked and confirmed the authenticity of the raw data.

\section{Ethics approval and consent to participate}

This study was approved by the Ethics Committee of Guizhou Medical University (Guiyang, China). Written informed consent was obtained from each patient.

\section{Patient consent for publication}

Not applicable.

\section{Competing interests}

The authors declare that they have no competing interests.

\section{References}

1. Lapointe S, Perry A and Butowski NA: Primary brain tumours in adults. Lancet 392: 432-446, 2018.

2. Chavda V,Patel V,Yadav D, Shah J,Patel S and Jin JO:Therapeutics and Research Related to Glioblastoma: Advancements and future targets. Curr Drug Metab 21: 186-198, 2020.

3. Qi Z and Chen L: Endoplasmic reticulum stress and autophagy. Adv Exp Med Biol 1206: 167-177, 2019.

4. Sehgal P, Szalai P, Olesen C, Praetorius HA, Nissen P, Christensen SB, Engedal N and Møller JV: Inhibition of the sarco/endoplasmic reticulum (ER) $\mathrm{Ca}^{2+}$-ATPase by thapsigargin analogs induces cell death via $\mathrm{ER} \mathrm{Ca}^{2+}$ depletion and the unfolded protein response. J Biol Chem 292: 19656-19673, 2017.

5. Chan CP, Kok KH and Jin DY: CREB3 subfamily transcription factors are not created equal: Recent insights from global analyses and animal models. Cell Biosci 1: 6, 2011.

6. Denard B, Jiang S, Peng Y and Ye J: CREB3L1 as a potential biomarker predicting response of triple negative breast cancer to doxorubicin-based chemotherapy. BMC Cancer 18: 813, 2018.

7. Liu LQ, Feng LF, Nan CR and Zhao ZM: CREB3L1 and PTN expressions correlate with prognosis of brain glioma patients. Biosci Rep: May 22, 2018 (Epub ahead of print).

8. Morishita S, Yasuda H, Yamawaki S, Kawaji H, Itoh M, Edahiro Y, Imai M, Kogo Y, Tsuneda S, Ohsaka A, et al: CREB3L1 overexpression as a potential diagnostic marker of Philadelphia chromosome-negative myeloproliferative neoplasms. Cancer Sci 112: 884-892, 2021.
9. Li XT, Tang W, Xie HJ, Liu S, Song XL, Xiao Y, Wang X, Cheng L and Chen GR: The efficacy of RGD modified liposomes loaded with vinorelbine plus tetrandrine in treating resistant brain glioma. J Liposome Res 29: 21-34, 2019.

10. Markouli M, Strepkos D, Papavassiliou AG and Piperi C: Targeting of endoplasmic reticulum (ER) stress in gliomas. Pharmacol Res 157: 104823, 2020.

11. Li Y, Ma H, Lu Y, Tan BJ, Xu L, Lawal TO, Mahady GB and Liu D: Menoprogen, a TCM herbal formula for menopause, increases endogenous E2 in an aged rat model of menopause by reducing ovarian granulosa cell apoptosis. Biomed Res Int 2016: $2574637,2016$.

12. Hassan M, Watari H, AbuAlmaaty A, Ohba Y and Sakuragi N: Apoptosis and molecular targeting therapy in cancer. Biomed Res Int 2014: 150845, 2014.

13. Penaranda Fajardo NM, Meijer C and Kruyt FA: The endoplasmic reticulum stress/unfolded protein response in gliomagenesis, tumor progression and as a therapeutic target in glioblastoma. Biochem Pharmacol 118: 1-8, 2016.

14. Song S, Tan J, Miao Y, Li M and Zhang Q: Crosstalk of autophagy and apoptosis: Involvement of the dual role of autophagy under ER stress. J Cell Physiol 232: 2977-2984, 2017.

15. Kondo S, Saito A, Asada R, Kanemoto S and Imaizumi K: Physiological unfolded protein response regulated by OASIS family members, transmembrane bZIP transcription factors. IUBMB Life 63: 233-239, 2011.

16. Vellanki RN, Zhang L and Volchuk A: OASIS/CREB3L1 is induced by endoplasmic reticulum stress in human glioma cell lines and contributes to the unfolded protein response, extracellular matrix production and cell migration. PLoS One 8: e54060, 2013.

17. Kondo S, Hino SI, Saito A, Kanemoto S, Kawasaki N, Asada R, Izumi S, Iwamoto H, Oki M, Miyagi $\mathrm{H}$, et al: Activation of OASIS family, ER stress transducers, is dependent on its stabilization. Cell Death Differ 19: 1939-1949, 2012.

18. Jaskulska A, Janecka AE and Gach-Janczak K: Thapsigarginfrom traditional medicine to anticancer drug. Int J Mol Sci 22: 4, 2020.

19. Wang H, Jia XZ, Sui CJ, Zhao YP, Mei YF, Zheng YN and Zhang ZY: Effects of thapsigargin on the proliferation and survival of human rheumatoid arthritis synovial cells. ScientificWorldJournal 2014: 605416, 2014.

20. Raiter A, Lipovetsky J, Hyman L, Mugami S, Ben-Zur T and Yerushalmi R: Chemotherapy controls metastasis through stimulatory effects on GRP78 and its transcription factor CREB3L1. Front Oncol 10: 1500, 2020.

21. Xiao W, Liang Y, Que Y, Li J, Peng R, Xu B, Wen X, Zhao J, Guan Y and Zhang X: Comparison of the MAID (AI) and CAV/IE regimens with the predictive value of cyclic AMP-responsive element-binding protein 3 like protein 1 (CREB3L1) in palliative chemotherapy for advanced soft-tissue sarcoma patients. J Cancer 10: 3517-3525, 2019.

22. Zhu HY, Bai WD, Liu JQ, Zheng Z, Guan H, Zhou Q, Su LL, Xie ST, Wang YC, Li J, et al: Up-regulation of FGFBP1 signaling contributes to miR-146a-induced angiogenesis in human umbilical vein endothelial cells. Sci Rep 6: 25272, 2016.

23. Cui X, Cui M, Asada R, Kanemoto S, Saito A, Matsuhisa K, Kaneko $\mathrm{M}$ and Imaizumi $\mathrm{K}$ : The androgen-induced protein AIbZIP facilitates proliferation of prostate cancer cells through downregulation of p21 expression. Sci Rep 6: 37310, 2016. 